\title{
EchoGéo
}

$44 \mid 2018$

Les valorisations territoriales et touristiques du street art

\section{Street art et mise en tourisme de la métropole parisienne, des festivals aux street art tours}

\section{Sophie Blanchard et Romain Talamoni}

\section{OpenEdition}

\section{Journals}

Édition électronique

URL : https://journals.openedition.org/echogeo/15663

DOI : 10.4000/echogeo. 15663

ISSN : 1963-1197

Éditeur

Pôle de recherche pour l'organisation et la diffusion de l'information géographique (CNRS UMR 8586)

Référence électronique

Sophie Blanchard et Romain Talamoni, «Street art et mise en tourisme de la métropole parisienne, des festivals aux street art tours », EchoGéo [En ligne], 44 | 2018, mis en ligne le 31 juillet 2018, consulté le 31 juillet 2021. URL : http://journals.openedition.org/echogeo/15663 ; DOI : https://doi.org/10.4000/ echogeo. 15663

Ce document a été généré automatiquement le 31 juillet 2021.

EchoGéo est mis à disposition selon les termes de la licence Creative Commons Attribution - Pas d'Utilisation Commerciale - Pas de Modification 4.0 International (CC BY-NC-ND) 


\title{
Street art et mise en tourisme de la métropole parisienne, des festivals aux street art tours
}

\author{
Sophie Blanchard et Romain Talamoni
}

1 L'éditeur de guides touristiques Lonely Planet a publié en 2017 un guide consacré au street art (Bartlett, 2017) ; Paris est en bonne place dans ce florilège des scènes street art des grandes métropoles mondiales. Le street art constitue donc désormais un élément incontournable de la visite urbaine, au sein d'une offre touristique métropolitaine qui se diversifie (Jacquot et al., 2013). Les visites street art permettent de rapprocher pratiques touristiques et pratiques habitantes de quartiers récemment rénovés (Maitland, 2013). Cette mise en tourisme du street art est contemporaine d'une institutionnalisation de la pratique alternative et transgressive du graffiti, inscrite dans la contre-culture. D'une part, l'intégration de la production des graffeurs au marché de l'art contemporain, par le biais de galeries et de marchands d'art, témoigne d'une forme de marchandisation de cette pratique artistique (Genard, 2014). D'autre part, le street art est mobilisé par les acteurs publics dans la production d'un art urbain qui s'intègre au modèle de la ville néo-libérale, dans le cadre d'une normalisation de la ville (Guinard et Margier, 2017). Cet art public se déploie notamment en parallèle du renouvellement urbain dans des quartiers ayant fait l'objet de grands projets urbains (Kullmann, 2015) et/ou en voie de gentrification (Blanchard, 2017).

2 Les pratiques touristiques et de loisir liées au street art dessinent une géographie particulière de la métropole parisienne. Elles participent à la mise en tourisme des quartiers périphériques de Paris intra-muros et de la proche banlieue, gentrifiés ou en cours de renouvellement urbain. Le street art contribue à construire une image de Paris comme métropole cosmopolite globalisée (Chapuis et Jacquot, 2014). Le lien entre l'institutionnalisation multiforme du street art et le développement de formes de tourisme métropolitain alternatif dans l'agglomération parisienne sera ici interrogé à travers l'exemple des street art tours, visites guidées payantes sur le thème du street art qui se sont développées très rapidement depuis une dizaine d'années à Paris et dans 
certaines communes de proche banlieue. En analysant les lieux et les acteurs de cette pratique touristique, nous interrogerons la façon dont les street art tours participent d'une recomposition des loisirs urbains.

3 Pour mener notre étude, nous avons participé à quatre visites organisées par différents acteurs, une à Vitry-sur-Seine et trois à Paris. Les visites parisiennes ont eu lieu dans le quartier de la Goutte d'Or, le long du canal de l'Ourcq, et dans le quartier de Belleville. Ces «balades» (terme le plus couramment employé par les organisateurs de street art tours pour désigner les visites, tant sur les sites internet qui les commercialisant que durant les visites elles-mêmes) ont été effectuées en 2015 pour la visite de Vitry, entre novembre et décembre 2017 pour les visites parisiennes. Elles ont été suivies d'entretiens avec deux organisateurs/guides.

4 Après avoir mis en évidence le développement de multiples pratiques de loisir liées au street art, nous verrons dans quelle mesure les street art tours s'intègrent à de nouvelles formes de tourisme métropolitain ciblant particulièrement les quartiers en cours de gentrification. Nous analyserons ensuite l'essor de ces nouvelles formes participatives de tourisme qui invitent à repenser les liens entre les artistes, les publics et les transformations de la métropole.

\section{Street art et pratiques de loisir, des festivals aux visites guidées}

\section{Les festivals et la normalisation du street art}

5 Le street art est aujourd'hui, dans les métropoles, support et prétexte de pratiques de loisir multiples, qui sont dans un premier temps liées explicitement à la dimension artistique du street art. Elles participent d'une offre de loisirs culturels en expansion dans la ville créative (Vivant, 2009), où l'art contribue au renouvellement urbain via la production d'une nouvelle image des quartiers. La notion de ville créative a été reprise par les milieux politiques (Keil et Boudreau, 2010) et largement intégrée à la gouvernance métropolitaine dans une optique de marketing urbain (Saez, 2014). Le street art peut alors être un outil (Blanchard, 2017; Sallenave, 2017) intégré aux politiques culturelles qui confortent le marketing urbain et favorisent la gentrification (Clerval et Fleury, 2009). D'abord valorisé au cours d'événements ponctuels, festivals et expositions, le street art est progressivement associé à des lieux culturels dédiés à la culture Hip Hop.

Expositions et festivals de street art commencent à émerger au début des années 2000 et se multiplient depuis. Dans le XIII ${ }^{e}$ arrondissement de Paris, la galerie Itinerrance a ainsi favorisé la création de fresques monumentales, puis a organisé, en 2013, une exposition éphémère dans une tour destinée à la démolition, la Tour Paris 13 (Kullman, 2015). Le long du canal de l'Ourcq, L'Été du canal, programme de festivités organisé depuis 2007 par le Comité départemental de la Seine-Saint-Denis, en partenariat avec les communes riveraines du canal, de Paris à Bondy, a mis le street art à l'honneur : en 2013, des photographies de l'artiste JR sont exposées le long des berges du canal (Exposition Inside Out); en 2014, un parcours street art intitulé De l'art à l'Ourcq est proposé, avec des croisières et des visites guidées. D'autres manifestations ponctuelles, tels les festivals de street art Ourcq Living Colors (depuis 2012) et le Festiwall (depuis 2016) ont émergé autour du canal, dans le XIX ${ }^{\mathrm{e}}$ arrondissement (Blanchard, 2017). Le 
long du canal Saint-Denis, c'est à l'occasion de l'Euro 2016 de football qu'est lancé le festival Street Art Avenue, fruit d'un partenariat entre acteurs publics (Plaine Commune, l'office de tourisme Plaine Commune Grand Paris, les villes de Paris, SaintDenis, Aubervilliers et le département de la Seine-Saint-Denis). Cette multiplication des événements street art dans l'espace public participe d'une forme de normalisation (Guinard et Margier, 2017) du street art dans le cadre de la ville créative. Selon R. Schacter (2014), il s'agirait même d'un processus de domestication, qui commence souvent par des festivals. Le street art est ainsi mis au service d'une politique culturelle de «festivalisation» par les collectivités locales (Sallenave, 2017), pour valoriser l'image de quartiers où le street art s'intègre aux pratiques de loisir. Lors de l'édition 2018 du Festiwall, le long du canal de l'Ourcq, les fresques réalisées ou en cours de réalisation ont ainsi attiré un public nombreux, mêlant familles du quartier et photographes amateurs.

Illustration 1 - Spectateurs du Festiwall, au bord du canal de l'Ourcq

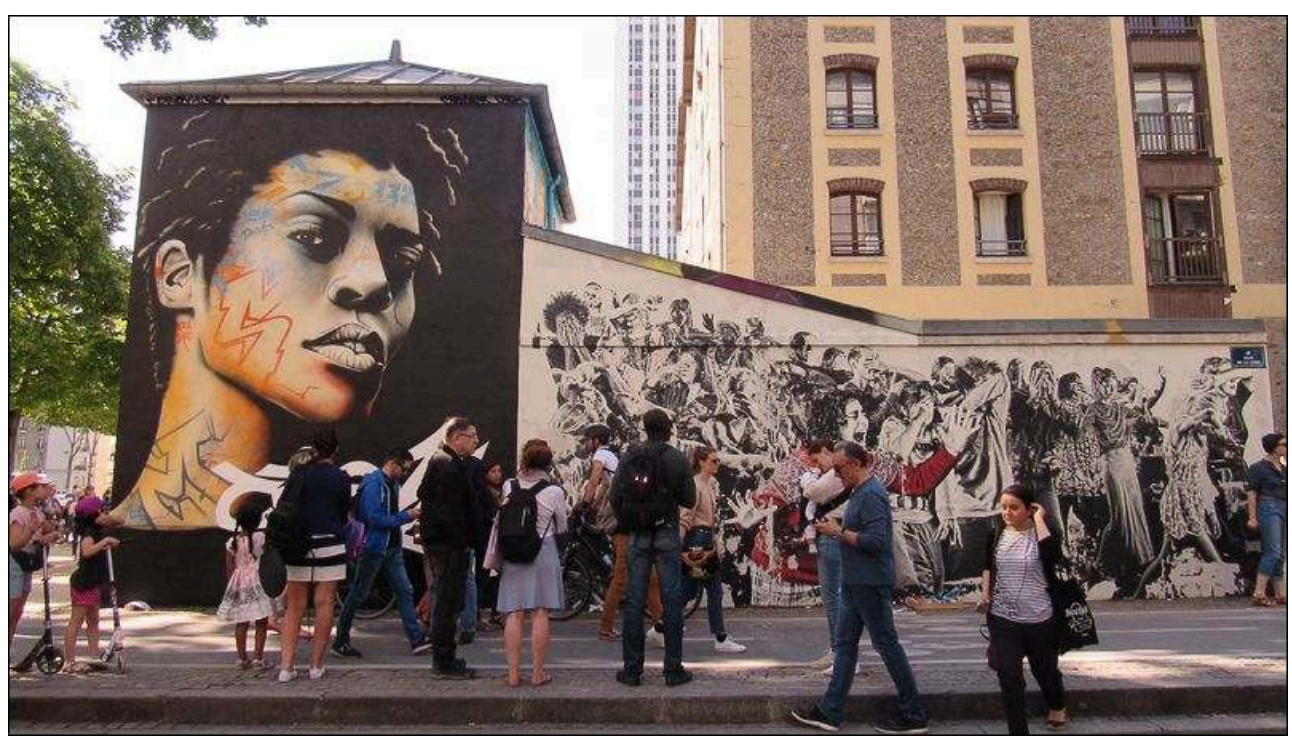

Auteur: S. Blanchard, 2018. 
Illustration 2 - Festiwall 2018, l'artiste à l'œuvre et son public

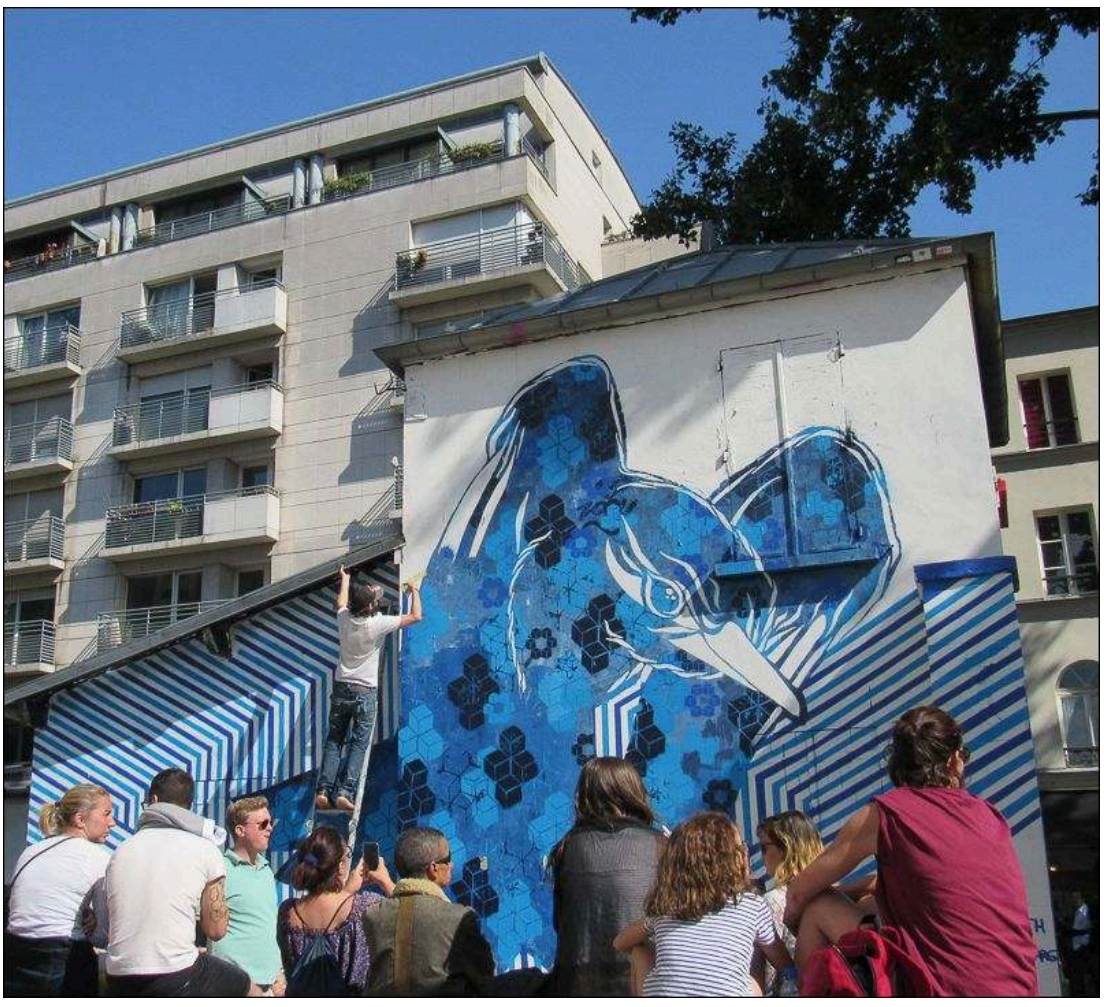

Auteur : S. Blanchard, 2018.

\section{Le street art, outil de l'institutionnalisation des cultures urbaines}

7 La fabrique d'un évènementiel fondé sur le street art s'inscrit dans un glissement plus large des cultures urbaines associées au Hip Hop, dont la dimension contestataire s'affaiblit à mesure qu'elles sont érigées en outil culturel et intégrées au marketing urbain. Cela est particulièrement notable dans le cas du street art: initialement le graffiti new-yorkais est lié à une forme de contestation sociale de l'ordre établi. Sa naissance est associée à des revendications ethniques et sociales propres au contexte étatsunien des années 1970 (Goldstein, 2009; Vulbeau, 1992). Son importation en Europe modifie sa portée; en effet, ceux qui le diffusent en France sont d'abord des artistes ayant séjourné aux États-Unis désireux d'explorer une forme d'art novatrice mais qui ne reprennent pas la dimension contestataire dans leur pratique. L'un d'entre eux, Philippe Lehman, dit Bando, issu d'une famille aisée parisienne, a démocratisé la technique du graffiti américain en France dans les années 1980 (Genin, 2013). Dès le départ, le graffiti perd donc une partie des revendications politiques qu'il portait dans les rues de New-York. Le graffiti, dans le contexte français, est importé par une élite. Il se démocratise dans un second temps et atteint les classes populaires. Sa diffusion en France, à partir de l'agglomération parisienne, dès les années 1980, a été d'emblée duale. Les tags vandales se sont multipliés alors que se développaient parallèlement des formes d'art urbain portées par des artistes, graphistes ou pochoiristes, comme Jérôme Mesnager ou Miss Tic, rapidement intégrés au marché de l'art (Genin, 2008) et à la gestion des espaces publics. Dès les années 1980 apparait, notamment dans la gestion de la SNCF, une distinction entre «mauvais tags » et «bonnes fresques» (Vulbeau, 1992). $\mathrm{Au}$ début des années 2000, le graffiti - le terme street art n'ayant pas encore été diffusé 
en France - est utilisé par les acteurs institutionnels pour toucher un public jeune issu des classes populaires. Le graffiti, et par extension le Hip Hop, sont alors un moyen d'intégrer ce public dans la programmation culturelle des villes de banlieue, comme à Vitry-sur-Seine (Talamoni, 2015). Les centres culturels qui proposent des ateliers d'initiation au graffiti, en collaboration avec des artistes dans des quartiers défavorisés, réalisent un travail social, l'artiste jouant également le rôle de médiateur (Milliot, 2000). Un élargissement de la portée des activités liées au street art, d'abord développées dans un but social, est alors perceptible: la culture Hip Hop, dans un premier temps associée à la politique de la ville, est peu à peu intégrée aux pratiques culturelles (Lafargue de Grangeneuve et al., 2008). Le projet Rosa Parks fait le mur, dans le XIX arrondissement de Paris, témoigne de cette volonté d'associer action sociale et valorisation culturelle (Blanchard, 2017).

8 L'ouverture par les collectivités locales et les entreprises publiques de lieux dédiés aux cultures urbaines témoigne de cette institutionnalisation. Dans le forum des Halles rénové, La Place, établissement culturel de la Ville de Paris dédié à la culture Hip Hop, a été inauguré au printemps 2016. Financé principalement par la mairie de Paris et appuyé par le département de Seine-Saint-Denis, cet espace participe de la construction d'une image de la métropole parisienne créative et capable d'intégrer les expressions artistiques venant des banlieues (Guillard, 2016). L'ouverture en 2017, dans le XVIII ${ }^{e}$ arrondissement, d'un espace de loisir éphémère consacré au street art, l'Aérosol, combine dynamique d'institutionnalisation et marchandisation du street art. L'Aérosol présente un ensemble de caractéristiques associées aux lieux « off »: la pratique du street art, encadrée ou non, l'emplacement, dans un entrepôt désaffecté de la SNCF dans le XVIII , le style et la décoration (les murs, les tables, les chaises et même le sol sont recouverts de graffitis de toutes natures, du simple tag à la fresque élaborée, le mobilier est peu abondant et dépareillé (Voir les illustrations 3 et 4)). Ce lieu associe une pratique alternative « domestiquée » à une activité commerciale. La SCNF, propriétaire de l'entrepôt est à l'initiative du projet, intégré plus largement à une politique d'urbanisme transitoire qui permet de créer des sites artistiques temporaires sur ses emprises en attente de reconversion urbaine. Elle y a intégré un magasin de fournitures pour graffeurs, Maquis-art, un bar et un musée, témoins d'une institutionnalisation du street art et d'une forme de marchandisation. Le site propose des locations pour des événements privés, avec une communication à destination des entreprises ${ }^{1}$. L'Aérosol peut donc être considéré comme un lieu emblématique d'une institutionnalisation de l'alternatif, impulsée par la diffusion de l'art urbain et intégrée aux pratiques de loisir métropolitaines. 
Illustration 3 - L'Aérosol, espace de loisir alternatif ou marchandisation du off?

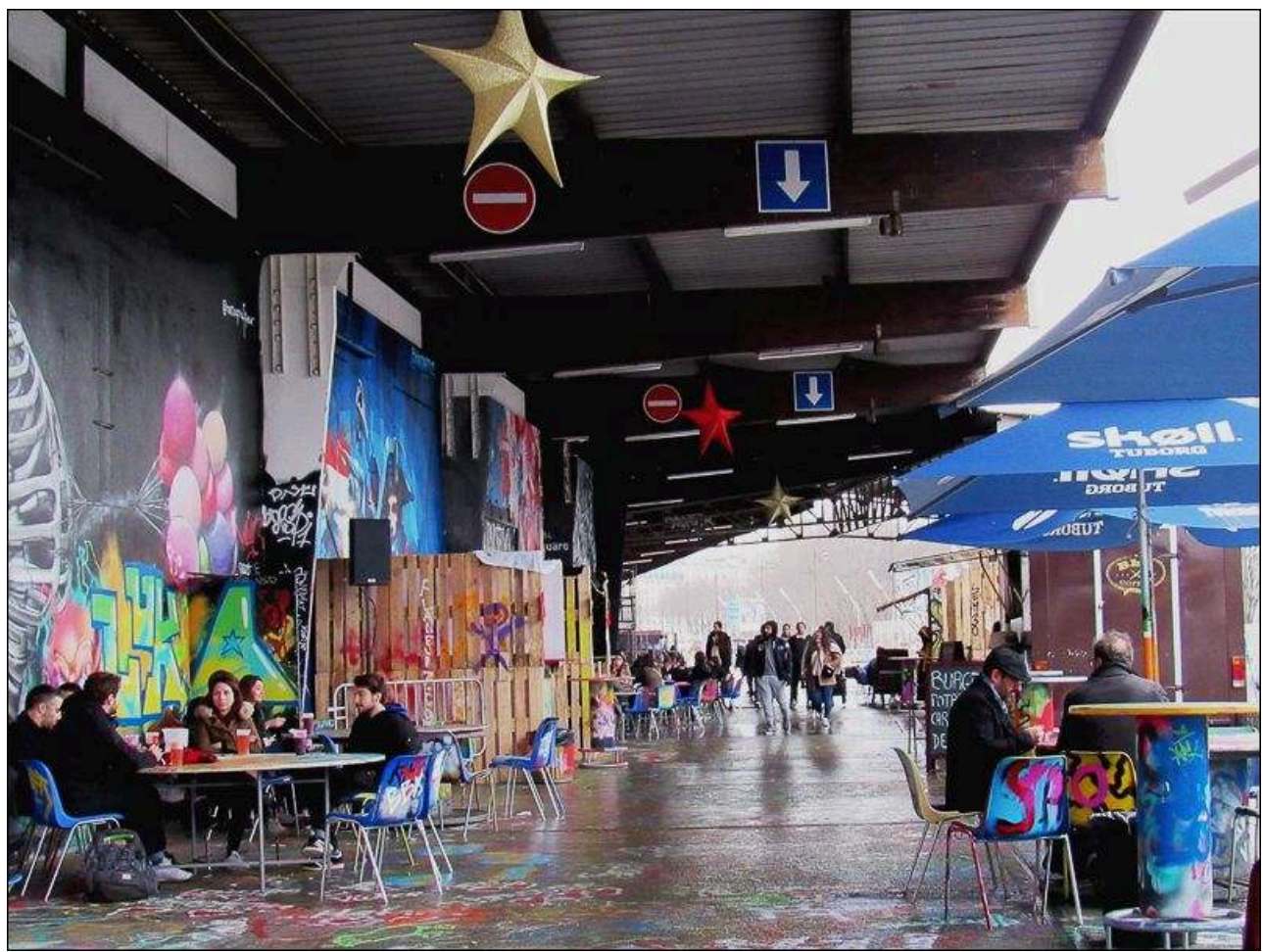

Auteur : S. Blanchard, 2017

Illustration 4 - L'Aérosol, espace de mise en scène de soi

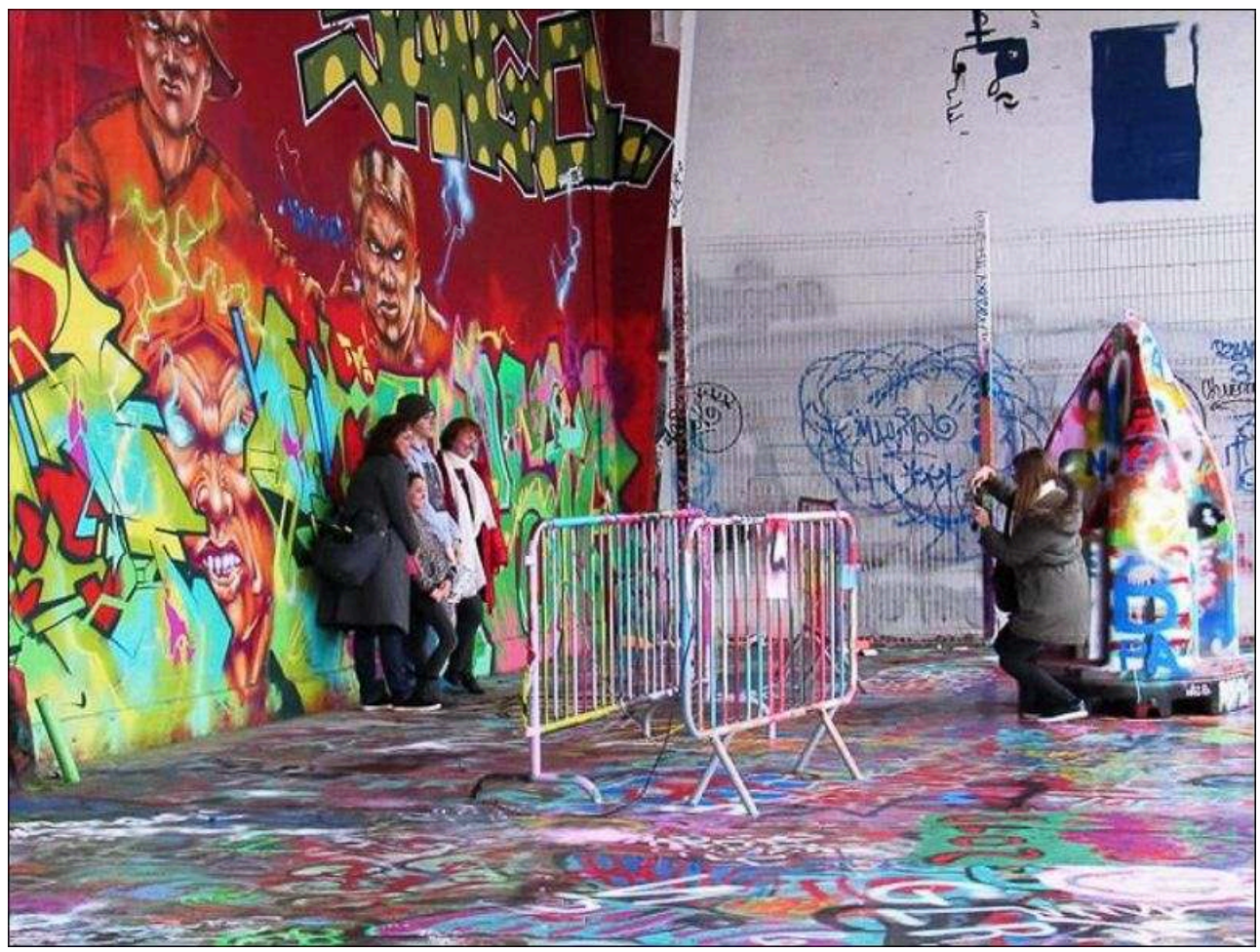

Auteur: S. Blanchard, 2017. 
Les acteurs du street art proposent en effet une multitude d'activités de loisir qui sont destinées à un public diversifié. Des ateliers didactiques à destination des enfants, des visites guidées pour les entreprises, des jeux d'orientation, autant d'activités qui renouvellent le lien entre street art et tourisme dans différents lieux. Si certaines activités de loisir fondées sur le street art ont une visée sociale et locale, une dynamique de création d'activités de loisir marchandes est nettement perceptible. La Street Dream Gallery, galerie dédiée au street art à Saint-Ouen, propose des ateliers d'initiation au graffiti pour les enfants en collaboration avec l'association Basalt, qui intervient également dans des écoles primaires du nord-est parisien. Le projet Street Art Up, initié par Hugo Benkara, a pour objectif de réaliser des fresques dans les entreprises. D'autres initiatives, comme le Street Art Express, proposent des jeux de pistes par équipe dont le but est de collecter, le long d'un parcours, des photos de fresque plus rapidement que les autres équipes. Ces trois exemples nous montrent que les pratiques de loisir fondées sur le street art sont variables en fonction des publics. L'institutionnalisation du street art lui avait ouvert les portes des galeries (Pradel, 2005), sa mise en tourisme le fait pénétrer dans des lieux tels que des bureaux ou des écoles et le présente dans des formes qui dépassent la simple exposition. Les expositions et festivals de street art sont aussi l'occasion de visites guidées ou street art tours qui attirent des touristes en quête d'art urbain et des habitants désireux de redécouvrir leur quartier. La particularité des street art tours est de proposer des activités à l'intersection du tourisme hors des sentiers battus et des loisirs métropolitains, tandis que les festivals cherchent à attirer des publics de connaisseurs mais également de curieux venant des aires métropolitaines élargies.

\section{Les street art tours, de l'insolite au tourisme ordinaire}

\section{La mise en tourisme d'espaces gentrifiés}

Quelle géographie de la métropole parisienne les balades street art dessinent-elles ? Les visites évitent les quartiers centraux du Paris patrimonialisé, à l'exception du Marais. Elles se concentrent dans les arrondissements périphériques et les communes de proche banlieue en voie de gentrification. Parmi les lieux les plus visités figurent le $\mathrm{XIII}^{\mathrm{e}}$ arrondissement, Belleville, Montmartre et le Marais: elles mettent donc à l'honneur surtout des espaces centraux et péricentraux précocement gentrifiés, dont l'image est ou a été associée à une contre-culture alternative et à la présence d'artistes off (Gravereau, 2013 ; Vivant et Charmes, 2008). Des espaces plus périphériques, dans Paris ou hors-les-murs, sont aussi fréquemment visités : les bords du canal de l'Ourcq et du canal Saint-Denis, Vitry-sur-Seine ou Montreuil. Le récent Guide du street art à Paris (Lombard, 2017) présente huit parcours sélectionnés (du Marais à Beaubourg; Mouffetard et la Butte aux Cailles; de République au 104 ; d'Oberkampf à Belleville ; Paris XIII, le pari des monumentales : autour de Montmartre; de Stalingrad à Pantin ; Vitry-sur-Seine, vitrine sur l'art urbain), en phase avec la géographie des visites ici décrites. Le guide Street Art (Lonely Planet), dans le chapitre consacré à Paris, mentionne les mêmes endroits (Bartlett, 2017). Les quartiers où se concentrent les visites connaissent ou ont connu des dynamiques de gentrification et/ou de renouvellement urbain. Les cas de figure divergent: le Marais, espace de gentrification ancienne au cœur du Paris patrimonialisé (Clerval, 2013), est emblématique d'une forme de 
centralité cosmopolite, tandis qu'à Vitry-sur-Seine la valorisation du street art participe davantage d'une dynamique de renouvellement urbain valorisant l'image de la «banlieue créative» (Talamoni, 2015). Les quartiers en cours de gentrification de la capitale, tels Belleville ou les abords du parc de La Villette, sont propices à l'évocation de la mémoire de la gentrification et à la présentation des dynamiques de changement urbain qui ouvrent aux street artists des espaces d'expression sur des bâtiments en attente de destruction ou de réaffectation. Au cours de nos visites, l'un des guides a par exemple évoqué une ancienne usine des chauffages parisiens, aujourd'hui détruite et remplacée par une supérette, support d'une des premières interventions collectives de l'artiste Da Cruz dans le quartier de l'Ourcq. Les références aux changements des quartiers visités et aux stratégies spatiales des street artists font partie de la scénographie des balades street art que nous avons suivies. La dynamique de gentrification est parfois explicitement évoquée. On peut ici faire l'hypothèse que les street art tours se développent alors dans la continuité des politiques culturelles qui accompagnent les processus de gentrification.

11 L'institutionnalisation du street art participe en effet de l'aménagement de la ville, via un embellissement des espaces publics qui accompagne - voire favorise, comme cela peut être le cas à Pantin (Blanchard, 2017) -, la gentrification (Clerval et Fleury, 2009) ; ce processus suivrait même des temporalités comparables à celles de la création de pistes cyclables et à la mise en avant de quartiers créatifs bohème (Schacter, 2014). La mise en avant du street art via une politique événementielle constitue un outil de marketing urbain, tant dans des quartiers de Paris ayant fait l'objet de projets urbains d'envergure comme le XIII ${ }^{\text {e }}$ arrondissement (Kullmann, 2015) que dans des communes de banlieue en cours de rénovation urbaine comme Pantin (Blanchard, 2017) ou Vitrysur-Seine (Talamoni, 2015). À travers la valorisation des arts urbains, les collectivités locales contribuent aussi à une forme d'institutionnalisation de l'alternatif (Daskalaki et Mould, 2013; Schacter, 2014), reprise par les acteurs privés qui organisent les visites: les termes "underground», "alternatif ", "cultures urbaines" sont ainsi mobilisés dans le langage promotionnel des associations et des entreprises qui proposent les balades. Ces acteurs vont cependant parfois bien au-delà de l'image stéréotypée du street art: pendant une visite à Belleville, une guide évoque ainsi largement l'histoire du mouvement graffiti, depuis les wagons de métro de New York dans les années 1980 jusqu'au graffuturism et au développement de nouvelles formes d'expression graphiques comme le calligraffiti, sans pour autant passer sous silence les enjeux de l'institutionnalisation et de la commande d'art urbain. Les street art tours ciblent donc des quartiers spécifiques à l'écart des circuits touristiques classiques (Gravari-Barbas et Delaplace, 2015) et permettent des formes originales de mise en tourisme.

\section{Les visites street art dans la mise en tourisme de la métropole : une marchandisation de l'exploration urbaine}

12 Le développement de formes alternatives de mise en tourisme contribue à transformer l'image de la métropole parisienne. La recherche de nouvelles formes de tourisme culturel et urbain, associée à une volonté de "sortir des sentiers battus " (GravariBarbas et Delaplace, 2015), est propice à une marchandisation de l'exploration urbaine. Les visites touristiques se sont fortement diversifiées depuis le début du XXI ${ }^{e}$ siècle, 
intégrant par exemple au circuit métropolitain des visites de quartiers d'immigration qui participent à la construction d'une "fable cosmopolite " (Chapuis et Jacquot, 2014). Le street art est désormais intégré à l'offre touristique de la métropole parisienne, comme en témoigne la publication d'un guide de promenades street art dans l'agglomération en 2017 (Lombard, 2017). Le tourisme «alternatif» fondé sur le street art a d'abord été lancé par des galeries, des artistes et des associations culturelles, dans une démarche à la fois promotionnelle et artistique. Outre des galeries désireuses de mettre en lumière leurs activités, des artistes ont aussi inventé des parcours permettant de découvrir leurs œuvres: le street artist Thom Thom a ainsi proposé plusieurs années durant des visites guidées le long du parcours De l'art à l'Ourcq. Les street art tours, inspirés par une pratique bénévole et spontanée d'exploration urbaine, se muent en une activité touristique, promue par les pouvoirs publics (via notamment le Comité départemental de Seine-Saint-Denis), visant à valoriser les espaces périphériques de la métropole (Jacquot et al., 2013). Ce type de pratique est en phase avec une mutation des activités de tourisme et de loisir tendant au brouillage des frontières entre habitants et touristes (Maitland, 2013).

Le succès rapide des street art tours a entraîné une diversification et une professionnalisation de l'offre de visites guidées sur le thème du street art, qui se sont multipliées dans différents quartiers de la capitale et de la proche banlieue. Les durées et les tarifs de ces visites varient : de 10 à 50 euros environ pour des balades de une à trois heures. La taille des groupes peut aller d'une demi-douzaine à une trentaine de personnes (Voir illustrations 5 et 6). Certaines visites sont centrées sur un artiste et peuvent inclure la visite d'un atelier, d'autres sont plus généralistes et restent dans l'idée de la découverte urbaine. Des visites spécialisées sont aussi proposées, en lien soit avec la pratique d'une activité artistique telle la photographie, soit avec un mode de déplacement, associées à la course à pied dans le cas des running street art tour $^{2}$ ou à la randonnée à bicyclette, plus rare (ce type d'offre semble beaucoup plus développée dans d'autres métropoles européennes telles Amsterdam ou Barcelone). L'association entre street art et pratique sportive manifeste ici une reconnaissance du street art comme un marqueur de l'identité urbaine et comme un élément moteur des loisirs urbains. Les street art tours ont aussi l'objectif de faire vivre à leurs clients une expérience urbaine incluant souvent une dimension participative. 
Illustration 5 - Groupe de visiteurs, rue Ordener

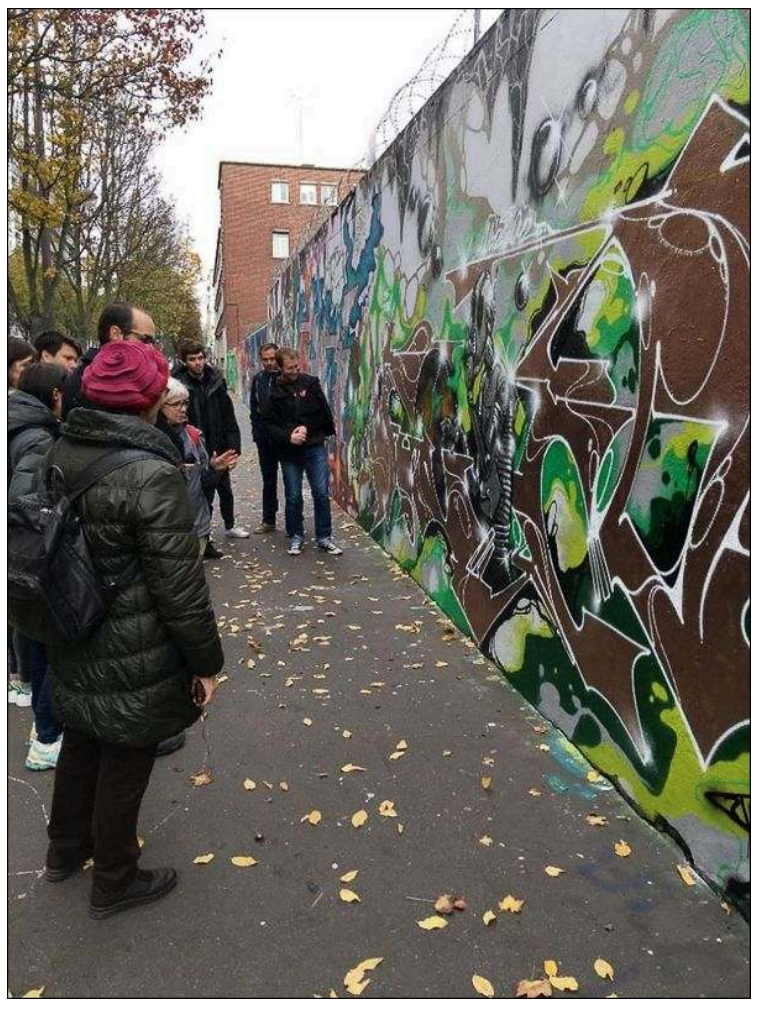

Auteur : R. Talamoni, 2017.

Illustration 6 - Groupe de visiteurs, rue Germaine Taillefer

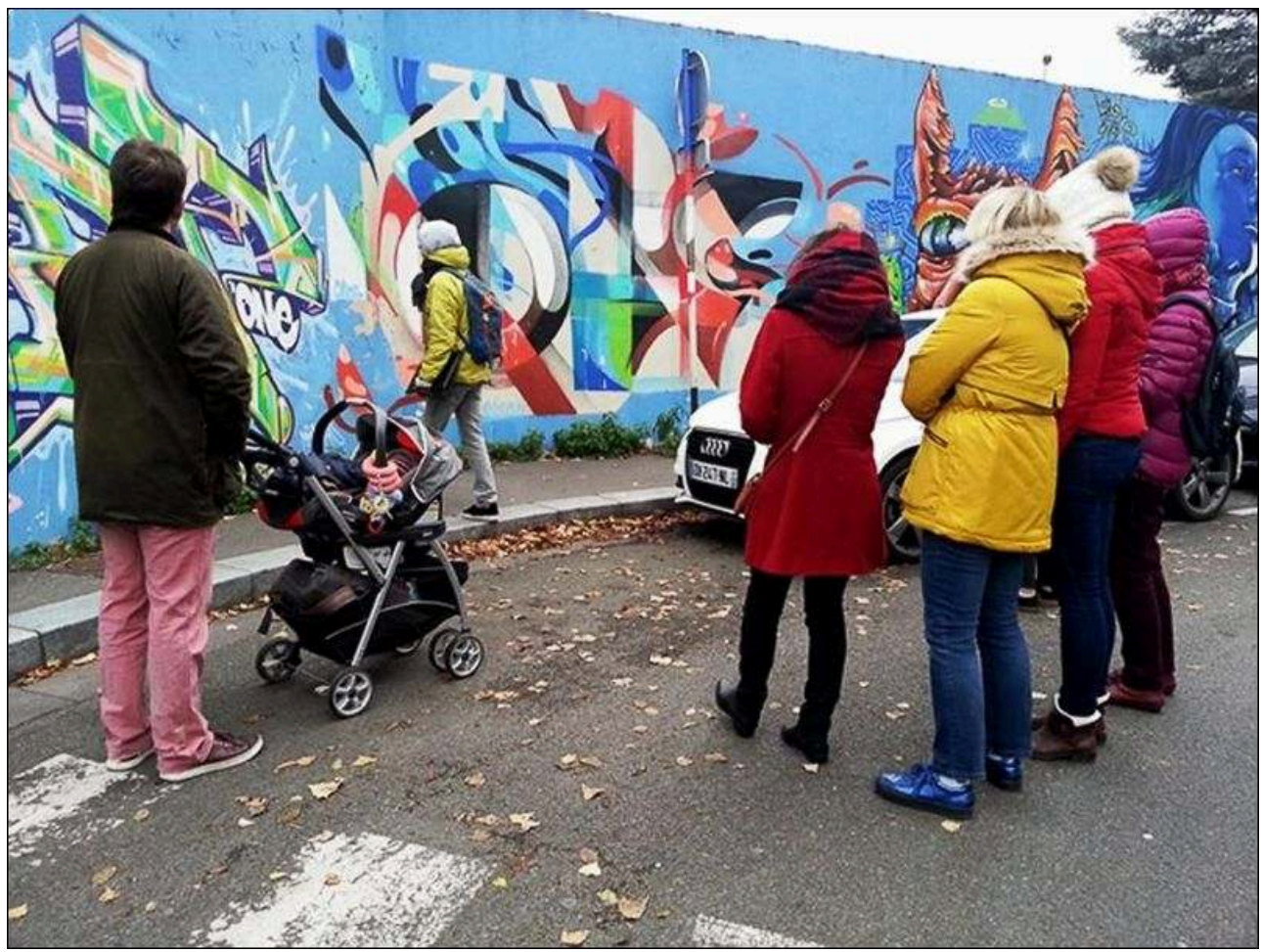

Auteur: S. Blanchard, 2017 


\title{
Repenser le lien entre les artistes et les publics : de nouvelles formes participatives de tourisme?
}

\author{
Le fonctionnement du street art tour : entre visite guidée et \\ exploration urbaine
}

Une des visites guidées retenue pour l'enquête était initiée par l'association Quai 36 qui valorise l'espace public et propose à ses usagers une « expérience esthétique, culturelle et humaine $»^{3}$. La promenade est également soutenue par le Comité départemental du Tourisme de Seine-Saint-Denis qui, depuis quelques années, promeut des initiatives similaires dans le nord-est parisien. Les réservations passent par la plate-forme en ligne du Comité et l'organisation du parcours est confiée à Quai 36 et Hugo Faivre, spécialiste de graffiti et organisateur de street art tour. La visite a lieu un samedi à 10 heures et le parcours débute à l'Institut des Cultures de l'Islam, au 19 rue Léon. Le guide attend les visiteurs dans le patio de l'institut, devant une fresque de Tarek Benaoum, artiste qui mêle la calligraphie arabe et le street art. Cette fresque a été soutenue et financée en partie par Quai 36, ce qui explique qu'elle constitue le point de départ de la visite. Le groupe est constitué de 25 visiteurs, il est assez hétérogène en termes d'âge et de genre ${ }^{4}$. Avant de se diriger vers la rue, le guide commence par un rapide historique du graffiti puis du street art. Il insiste sur la terminologie et les différences entre le tag, le graff et le street art. Le public est intéressé et curieux dans l'ensemble, parfois connaisseur. Les noms d'artistes évoqués par le guide font quelquefois écho chez les participants à d'autres visites. Le groupe s'engage rue Léon, il la remonte vers le nord. Le guide souhaite nous emmener rue Ordener qui abrite le «plus long mur de graffitis d'Europe $»^{5}$. Sur le parcours, Hugo Faivre marque des pauses devant des rideaux métalliques de magasins et des camions peints par des graffeurs, des tags de $c r e w^{6}$ que le guide connaît personnellement, des pochoirs de street-artists, des mosaïques, des moulages, des détournements de signalisation... autant de marquages que les visiteurs ne perçoivent pas forcément quand ils pratiquent l'espace urbain. Le parcours est donc agrémenté d'anecdotes sur les artistes et la conception des fresques, les visiteurs pouvant questionner le guide afin d'enrichir leur connaissance sur la discipline. La visite se poursuit rue Ordener, qualifiée par le guide de véritable «musée à ciel ouvert ». Les participants, admiratifs, avancent le long du mur, prennent des photos et commentent les fresques. Le groupe, statique sur le trottoir, contraint les passants à descendre sur la route pour le contourner. Après avoir descendu le boulevard Barbès, la visite se poursuit le long du boulevard de la Chapelle où des mosaïques de Space Invader sont visibles sur les piliers du métro aérien. Enfin, le groupe rejoint la Gare du Nord, où des fresques ont été financées par Quai 36 : celles de l'artiste Bebar, rue de Maubeuge, et celles de divers artistes sur la voie 36 des lignes régionales de la gare.

\section{Rencontrer et participer : replacer le touriste au cœur de l'expérience artistique}

15 Le projet d'Hugo Faivre ${ }^{7}$, organisateur de street art tours et fondateur de Background Paris, a pour objectif de présenter la ville de Paris à travers différentes disciplines artistiques issues du Hip Hop, à savoir la danse, le rap et le graffiti. Le fonctionnement des street art tours est semblable à celui d'une visite au musée : un guide partage son 
savoir sur des œuvres avec un public. Durant les promenades, les guides présentent les fresques en donnant aux visiteurs des clefs de lecture pour comprendre la démarche des artistes. Ce format pose plusieurs questions relatives à la propriété des œuvres, étant donné que la majeure partie des visites ont lieu dans l'espace public. L'appartenance des œuvres n'est pas clairement définie, l'illégalité de l'acte et la propriété des murs ne permettant pas à l'artiste de jouir de droits d'auteur. En effet, le vol récent des œuvres de l'artiste Space Invader ${ }^{8}$ a mis en évidence le flou juridique concernant la propriété des fresques. Les artistes ne participent pas, dans la plupart des cas, à l'élaboration des promenades et ne perçoivent pas de rémunération.

Hugo Faivre déplore le manque de lien entre les artistes, les publics et les guides, et préconise la rencontre entre ces trois groupes pour repenser la manière de concevoir les promenades. Après avoir travaillé dans des galeries en tant que commissaire d'exposition de street art, Hugo Faivre a voulu penser un format d'exposition qui permet à la fois de mettre en valeur un espace et des artistes par le tourisme. Pour cela il s'est associé avec la plate-forme de logement Airbnb ${ }^{9}$. Le public ciblé par Hugo Faivre est constitué de touristes voulant agrémenter leur visite de Paris d'une expérience originale et désireux de voir les coulisses de la scène street art parisienne (Maitland, 2013). Pour le touriste, la participation à ce type d'activité est précieuse puisqu'elle est éphémère. D'après E. Vivant, la temporalité limitée de ces visites est recherchée car le touriste se sent le témoin privilégié d'un événement singulier. Les pratiques culturelles off sont pour le touriste un moyen de rendre son voyage unique, d'autant plus que certains lieux off seraient menacés par la gentrification (Vivant, 2009).

Je me suis demandé comment présenter le travail d'un artiste sans avoir le prisme de la vente : en proposant des rencontres entre le public et les artistes. Lorsqu'on fait un voyage, ce dont on se souvient, c'est la rencontre. Si je vais à New York et que j'y rencontre un writer new-yorkais, quand je vais revenir à Paris, je vais me souvenir de cette rencontre-là plus que d'une paire de basket que j'ai achetée » (Hugo Faivre, entretien du 11/12/2017, Centre La Place).

17 La rencontre entre les artistes et le public est un moyen de rendre ces visites participatives. Dans le cas des promenades organisées par Hugo Faivre, les rencontres se font in situ, dans l'atelier de l'artiste ou sur un mur choisi par ce dernier. Cela permet à l'artiste de montrer son processus créatif et aux touristes de s'essayer à certaines techniques du street art. Ainsi, Hugo Faivre tente de se démarquer des visites guidées traditionnelles et propose une expérience touristique qu'il veut plus « authentique ».

\section{Conceptions divergentes et conflits d'appropriation}

18 Les acteurs qui participent à la mise en tourisme du street art ne partagent pas les mêmes objectifs. La participation des publics et la rencontre avec les artistes ne peuvent être possibles que sous certaines conditions. Cette manière de concevoir la promenade nécessite la constitution de petits groupes de touristes pour permettre une proximité et un échange avec l'artiste. Au sujet des autres acteurs effectuant des street art tours, Hugo Faivre n'emploie pas le terme de concurrence, néanmoins il insiste sur le fait que leur format de visite ne permet pas une expérience participative et immersive pour le visiteur.

J'essaie de me démarquer d'eux, parce qu'on se croise tous les dimanches dans le $\mathrm{XIII}^{\mathrm{e}}$ avec nos groupes respectifs. Pour avoir cette intimité avec l'artiste, je ne peux pas venir dans un atelier avec trente personnes, donc maximum dix personnes : par 
expérience, on [ne] peut pas réserver plus. Je les ai croisés l'autre fois, nous on était quatre et, eux, ils étaient 36 quoi ! Comment tu veux faire une promenade avec autant de monde? » (Hugo Faivre, entretien du 11/12/2017, Centre La Place). qui pratiquent l'espace visité. Durant une promenade organisée par une association à Vitry-sur-Seine en 2015, des frictions ont pu être observées entre un groupe de visiteurs et de jeunes habitants d'une cité jouant au basket-ball sur un terrain bordé par des fresques de graffiti (Voir illustration 7). En effet, le passage des visiteurs sur le terrain a interrompu de manière temporaire le match, provoquant des réactions assez vives de la part des jeunes. Les visiteurs sont ici considérés comme des étrangers qui pénètrent dans un espace qui n'est pas le leur, pour prendre en photo des fresques qui ne leurs appartiennent pas. Les jeunes manifestent leur étonnement et leur stupeur et expliquent que cette visite est perçue comme une intrusion: "Vous faites quoi ici? Vous n'êtes pas chez vous!». Les touristes, venant en majorité de la ville de Paris, poursuivent leur activité sans prêter garde aux invectives des jeunes, le guide les dirigeant ensuite vers une autre fresque plus isolée. La présence de ces touristes dans des espaces à l'écart des circuits touristiques traditionnels questionne l'impact sur les quartiers visités de ces street art tours. Pour le guide de cette association, la visite est un moyen pertinent de renverser le stigmate des villes de banlieue et de donner à voir l'aspect créatif et artistique de Vitry-sur-Seine. Cependant, valoriser ces espaces par le street art peut amener à occulter les réalités sociales de ceux-ci. Les exemples précédemment évoqués montrent bien des logiques de concurrences entre les acteurs de la mise en tourisme du street art; des conflits d'appropriation surviennent au sein d'espaces touristiques convoités par de multiples acteurs, notamment lorsque les manières de concevoir la pratique de ce tourisme divergent. Cela peut aussi être le signe d'un rapport de domination entre des touristes à la recherche d'une authenticité et des habitants de quartiers défavorisés, dans le cadre d'une mise en tourisme élargie des aires métropolitaines. 


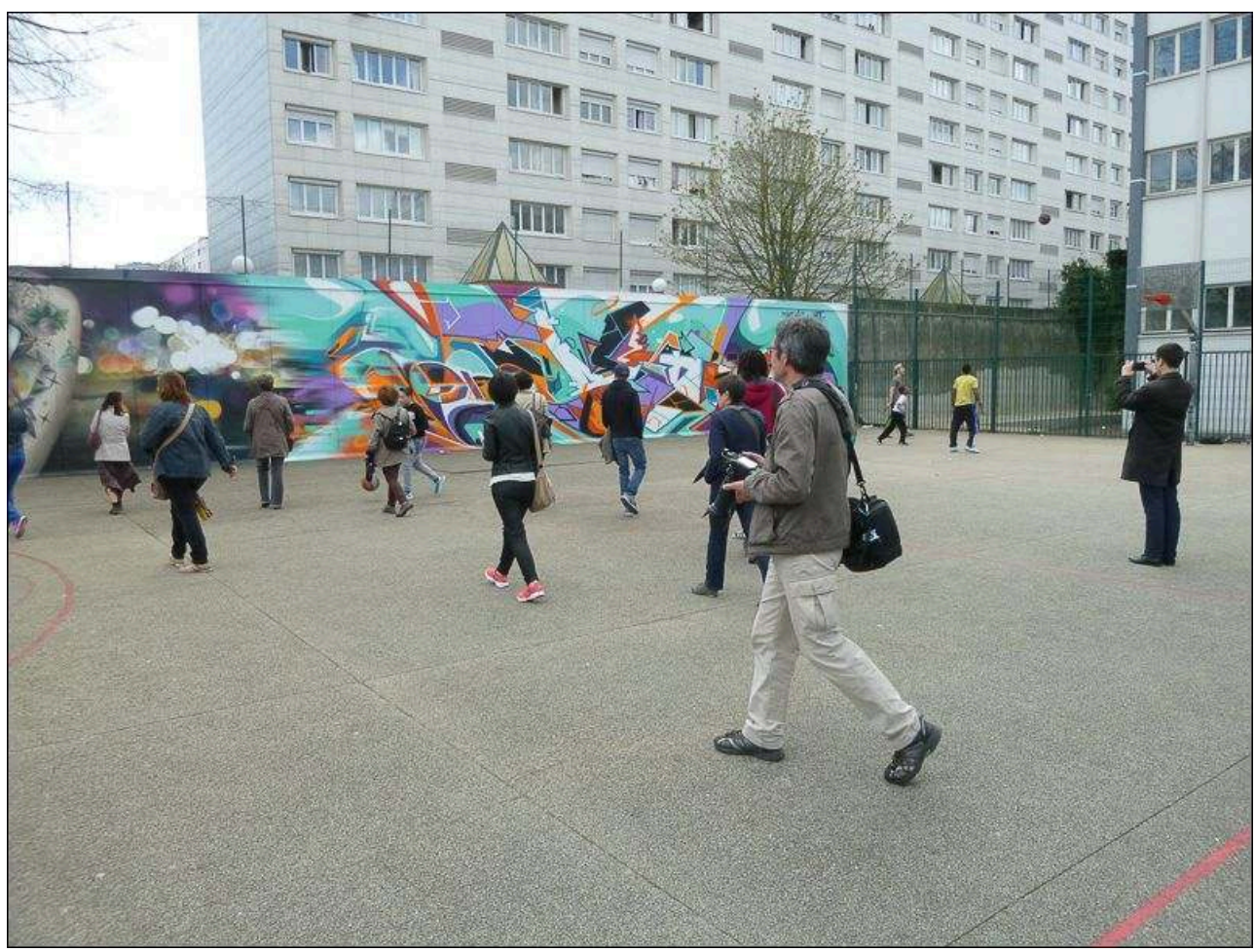

Auteur : R. Talamoni, 2015.

21 Ces réflexions nous invitent à de nouveaux questionnements sur les processus de catégorisation et de domination induits par la mise en valeur du street art. La mise en tourisme du street art accompagne la gentrification et participe au changement d'image d'un quartier ou d'une ville. Le street art n'est pas un moteur du processus, il est le marqueur d'une appropriation temporaire de l'espace par des visiteurs très divers: habitants en quête d'un nouveau regard sur leur quartier, élèves des établissements scolaires du quartier, touristes qui ne pratiquent pas le parcours de la promenade dans leur quotidien... Cela peut engendrer des conflits entre visiteurs et habitants, voire des résistances des habitants face à la mise en tourisme de leur quartier. Le tourisme met en effet en jeu des rapports de pouvoir, de domination et de résistance (Boukhris et Chapuis, 2016) qui régissent également le champ du street art. Le tourisme contribue à augmenter le capital symbolique d'un espace, les parcours street art revalorisant certains quartiers de la métropole. Les festivals, quant à eux, s'inscrivent dans une optique plus large de marketing urbain souvent pensée à l'échelle des métropoles (Sallenave, 2017). À plus grande échelle, une fresque assigne une identité créative à un pan de mur, un recoin ; elle peut être utilisée pour entraîner l'éviction de certaines populations considérées comme indésirables. M. Froment-Meurice décrit ainsi comment, lors d'un conseil de quartier parisien, il a été décidé de commander une fresque street art pour habiller un pan de mur devant lequel étaient installés des sansabri, la fresque faisant ici figure de méthode " douce " pour évincer les indésirables (Froment-Meurice, 2016, p. 334).

Le street art s'inscrit donc dans un jeu d'inégalités et de rapports de pouvoir. Une activité touristique qui a lieu dans l'espace public n'est en effet pas ouverte à tous, sa gratuité et son accessibilité n'étant pas garants de son ouverture. Des inégalités 
s'observent aussi bien parmi les acteurs mettant en valeur le street art par le tourisme que parmi les visiteurs. Des inégalités de genre premièrement, du fait d'une sousreprésentation des femmes chez les organisateurs de street art tours et chez les artistes mis en valeur durant les parcours. Des inégalités sociales, même si celles-ci sont difficilement perceptibles : vu le coût des visites et les stratégies promotionnelles mises en place par les acteurs des street art tours, nous pouvons supposer que les publics des visites street art possèdent un capital culturel élevé et appartiennent à des classes sociales moyennes ou supérieures. Il serait intéressant d'approfondir ces questionnements sur les publics, afin de voir si le street art, dans sa dimension récréative, s'ouvre à tous les types de public ou bien s'il reproduit des inégalités sociales. On peut alors postuler l'existence d'un gradient de distinction au sein même des activités fondées sur le street art qui mêlent action à but social et pédagogique, promenades libres inscrites dans les loisirs métropolitains au sein de quartiers en mutation et offre touristique marchande institutionnalisée.

\section{BIBLIOGRAPHIE}

Bartlett E., 2017. Street Art (Lonely Planet). Lonely Planet, 224 p.

Blanchard S., 2017. Street art, rénovation urbaine et gentrification dans le Nord-Est parisien : entre marketing urbain et gender mainstreaming. Urbanités [En ligne], n9. http://www.revueurbanites.fr/9-street-art-renovation-urbaine-et-gentrification-dans-le-nord-est-parisien-entremarketing-urbain-et-gender-mainstreaming/

Boukhris L., Chapuis A., 2016. Circulations, espace et pouvoir. Penser le tourisme pour penser le politique. L'Espace Politique [En ligne], $\mathrm{n}^{\circ} 28$. http://journals.openedition.org/espacepolitique/ 3707

Chapuis A., Jacquot S., 2014. Le touriste, le migrant et la fable cosmopolite. Mettre en tourisme les présences migratoires à Paris. Hommes et Migrations, n 1308, p. 75-86.

Clerval A., Fleury A., 2009. Politiques urbaines et gentrification, une analyse critique à partir du cas de Paris. L'Espace politique [En ligne], $\mathrm{n}^{\circ}$ 8. http://journals.openedition.org/espacepolitique/ 1314

Clerval A., 2013. Paris sans le peuple. La gentrification de la capitale. Paris, La Découverte, 255 p.

Daskalaki M., Mould O., 2013. Beyond Urban Subcultures: Urban Subversions as Rhizomatic Social Formations. International Journal of Urban and Regional Research, vol. 37, nº 1, p. 1-18.

Froment-Meurice M., 2016. Produire et réguler les espaces publics contemporains : les politiques de gestion de l'indésirabilité à Paris. Thèse de doctorat en Géographie, Université Paris-Est, 388 p.

Genard J.-L., 2014. Cultures urbaines et politiques culturelles. SociologieS, Diversification artistique et politiques culturelles [En ligne]. http://journals.openedition.org/sociologies/4575

Genin C., 2013. Le street art au tournant. Reconnaissance d'un genre. Bruxelles, Les Impressions nouvelles, $272 \mathrm{p}$. 
Genin C., 2008. Miss Tic : Femme de l'être. Bruxelles, Les Impressions nouvelles, 208 p.

Goldstein R., 2009. Street and self. Les origines du graffiti. In Fondation Cartier pour l'art contemporain. Né dans la rue, Graffiti, Paris, Fondation Cartier, p. 4-6.

Gravari-Barbas M., Delaplace M., 2015. Le tourisme urbain « hors des sentiers battus ». Téoros [En ligne], $\mathrm{n}^{\circ}$ 34. http://journals.openedition.org/teoros/2790

Gravereau S., 2013. Les artistes de Belleville : valeur et faire-valoir d'un quartier de Paris à leurs dépens? Territoire en mouvement Revue de géographie et aménagement [En ligne], $\mathrm{n}^{\circ}$ 17-18. http:// tem.revues.org/1999

Guillard S., 2016. Musique, villes et scène : localisation et production de l'authenticité dans le rap en France aux États-Unis. Thèse de doctorat en géographie, Université Paris-Est, 521 p.

Guinard P., Margier A., 2017. Art as a new urban norm: Between normalization of the City through art and normalization of art through the City in Montreal and Johannesburg. Cities, [En Ligne]. http://dx.doi.org/10.1016/j.cities.2017.04.018

Jacquot S., Gravari-Barbas M., Fagnoni E., 2013. Patrimonialisation et tourisme dans la région métropolitaine parisienne. Le patrimoine, clé de métropolité touristique. In M. Gravari-Barbas M., et F. Fagnoni, Métropolisation et tourisme. Paris, Belin, p. 103-117.

Keil R., Boudreau J-A., 2010. Le concept de la ville créative : la création réelle ou imaginaire d'une forme d'action politique dominante. Métropoles [En ligne], $\mathrm{n}^{\circ}$ 7. http://journals.openedition.org/ metropoles/4339

Kullmann, C., 2015. De l'exposition de la Tour Paris 13 au concept de musée à ciel ouvert. Téoros [En ligne], n³4. http://journals.openedition.org/teoros/2776

Lafargue de Grangeneuve L., Kauffmann I., Shapiro R., 2008. Cultures urbaines, territoires et action publique. Rapport final pour le ministère de la Culture et de la Communication, Paris, 168 p.

Lombard S., 2017. Guide du street art à Paris. Paris, Alternatives, 140 p.

Maitland R., 2013. Backstage Behaviour in the Global City: Tourists and the search of the 'real London'. Procedia. Social and Behavioral Sciences, $n^{\circ} 105$, p. 12-19.

Milliot V., 2000. Quand l'art interroge l'espace public. Le graff, le travail social, l'art contemporain et le politique. In J.P. Saez, T. Raspail (dir.), L'art contemporain, champs artistiques, critères, réception. Actes du colloque l'Art sur la Place. Paris, L'Harmattan, p. 179-195.

Pradel B., 2005. Entre institutionnalisation et clandestinité : le graffiti ou l'hydre à deux têtes. Consommations et sociétés, $\mathrm{n}^{\circ} 7, \mathrm{p} .177-189$.

Saez G., 2014. La métropolisation de la culture. Cahiers français, $n^{\circ} 382$, p. 10-15.

Sallenave L., 2017. Le « Grenoble Street Art Fest » catalyseur d'images institutionnalisées et détournées. Enjeux discursifs et territoriaux. Urbanités [En ligne], $\mathrm{n}^{\circ}$ 9. http://www.revueurbanites.fr/9-le-grenoble-street-art-fest-catalyseur-dimages-institutionnalisees-et-detourneesenjeux-discursifs-et-territoriaux/

Schacter R., 2014. The ugly truth: Street Art, Graffiti and the Creative City. Art \& the Public Sphere, vol. 3, $\mathrm{N}^{\circ}$ 2, p. 161-176.

Talamoni R., 2015. Les stratégies de mise en valeur du « treet art » à Vitry-sur-Seine. Mémoire de Master 2, UPEC, $110 \mathrm{p}$.

Vivant E., 2009. Qu'est-ce que la ville créative ? Paris, Presses Universitaires de France, 96 p. 
Vivant E., Charmes E., 2008. La gentrification et ses pionniers : le rôle des artistes off en question. Métropoles [En ligne], n 3. http://metropoles.revues.org/1972

Vulbeau A., 1992. Du tag au tag. Malakoff, Desclée de Brouwer, 110 p.

Vulbeau A., 1992. Les tags, spectre de la jeunesse : Histoire d'une nouvelle pratique urbaine. Les Annales de la Recherche Urbaine, $n^{\circ}$ 54, p. 61-67.

\section{NOTES}

1. http://www.laerosol.fr/2-privatisations.php

2. https://www.tourisme93.com/visites/fr/2716-running-tour-street-art-sur-le-canal-saintdenis.html

3. http://quai36.com/fr/

4. Une enquête quantitative aurait pu permettre de dégager un profil sociologique poussé du groupe mais elle aurait limité l'immersion de l'observation participante et aurait nécessité un entretien préalable avec le guide.

5. https://www.institut-cultures-islam.org/agenda/piste-street-art-visite-quai-36/

6. Collectif de graffeurs qui possède un tag identifiable par les autres collectifs souvent sous forme d'acronyme.

7. Hugo Faivre est un autoentrepreneur qui travaille au sein de l'incubateur d'entreprises du centre La Place.

8. Des mosaïques du street-artist français Space Invader ont été dérobées dans Paris en août 2017 par deux individus. La mairie de Paris a décidé de porter plainte pour usurpation d'identité car les voleurs se sont fait passer pour des agents d'entretien municipaux.

9. La plate-forme Airbnb propose aux touristes des expériences en plus de leurs logements. Ces expériences peuvent être des performances d'artistes, des dîners, des projections...

\section{RÉSUMÉS}

Le street art est l'objet de formes de mise en tourisme dans la métropole parisienne. À Paris et en proche banlieue, à la suite de l'émergence d'expositions et de festivals de street art, des visites guidées sur le thème du street art se sont multipliées. Ces promenades urbaines, souvent présentées sous l'appellation de street art tours, mobilisent des acteurs privés et visent un public diversifié, des Parisiens curieux aux touristes passant par la plate-forme de logement Airbnb. Les street art tours contribuent à la mise en tourisme des quartiers gentrifiés ou en rénovation de la métropole, tout en s'inscrivant dans des rapports de pouvoir entrecroisés.

In the Parisian metropolis, street art is becoming a touristic object. It started with festivals and art exhibitions, followed by an increasing number of guided street art tours, in Paris and the suburbs. The private actors who organize these street art tours try to reach a very large audience, from curious locals to international tourists, and use mainly the internet for their promotion, via independent sites as well as major ones like Airbnb. Street art tours participate in the touristification of gentrified or renovated areas, therefore taking part in the intertwined power relations of urban society. 
INDEX

Keywords : Gentrification, leisure, metropolis, street art, tourism

Mots-clés : gentrification, loisirs, métropole, street art, tourisme

\section{AUTEURS}

\section{SOPHIE BLANCHARD}

Sophie Blanchard, sophie.blanchard@u-pec.fr, est PRAG à l'Université Paris-Est-Créteil, membre de Lab'Urba. Elle a publié récemment :

- Blanchard S., 2017. Street art, rénovation urbaine et gentrification dans le Nord-Est parisien : entre marketing urbain et gender mainstreaming. Urbanités, $\mathrm{n}^{\circ} 9$, [En Ligne] http://www.revueurbanites.fr/9-street-art-renovation-urbaine-et-gentrification-dans-le-nord-est-parisien-entremarketing-urbain-et-gender-mainstreaming/

- Blanchard S., 2014. Intersectionnalité, migrations et travail domestique : lectures croisées en France et aux États-Unis. Echogéo, n³0, Rubrique Sur l'Ecrit, [En Ligne] http://

echogeo.revues.org/14073

\section{ROMAIN TALAMONI}

Romain Talamoni, romain.talamoni@ac-creteil.fr, est professeur agrégé de géographie au lycée Georges Brassens à Villeneuve-le-Roi. 